\title{
ASYMMETRIC DIRECTIONAL COUPLER CONTROLLED BY PHOTOREFRACTIVE GRATING
}

\author{
E. WEINERT-RączKa* and R. IWANOW \\ Institute of Electronics and Informatics, Technical University of Szczecin \\ Al. Piastów 17, 70-310 Szczecin, Poland
}

An asymmetric three channel directional coupler with a thin photorefractive grating as an all-optical switching and wavelength demultiplexing element with memory is analysed.

PACS numbers: 42.65.-k, 42.65.Pc

\section{Introduction}

- Transfer of light between different waveguides is one of the crucial functions of the integrated optics systems. The power exchange between waveguides, placed in a closed proximity to form a directional coupler, is efficient when the light propagates in them with the same velocity. In the case of different propagation constants the coupling can be obtained by means of a diffraction grating formed by periodic changes of the refractive index along the propagation direction [1-5]. The grating constant $K=2 \pi / \Lambda$ (where $\Lambda$ is the length of the grating period) should be equal to the difference between propagation constants of guided modes ( $K=$ $\beta_{A}-\beta_{B}$ ). Such systems, called grating assisted couplers (GACs) are used as narrow frequency filters and demultiplexing elements. The grating in the traditional GAC is formed during the fabrication process and has constant, immutable parameters, which limits the operation of the coupler to one, strictly defined frequency. A tuneable coupler can be realised when the grating is created by two external beams interfering in a nonlinear medium [6]. Parameters of such grating depend on the external waves properties and can be varied during the work of the device. In particular the grating constant can be tuned to the guided modes propagation constants difference by changing the external waves frequencies or propagation directions.

Here we propose a new type of the directional coupler which is controlled by the photorefractive grating. The grating is created by a short pulse of the external waves and lasts until the arrival of the next pulse which can amplify or erase it. Guided modes being the subject of switching have much lower frequencies and their influence on the grating parameters is negligible.

*e-mail: ewar@arcadia.tuniv.szczecin.pl 


\section{Performance of the coupler}

The coupler consisting of three different channel waveguides parallel to the $z$-axis of the coordinate system is presented in Fig. 1. Parameters of the channels differ enough to avoid power exchange between them. The refractive index grating, required to mediate coupling, is created by two external waves forming an interference pattern along the $z$ axis

$$
I(x, y, z, t)=I_{0}(x, z, t) \exp \left[\alpha_{\mathrm{ex}}\left(y-y_{0}\right)\right][1+m \cos (K z)],
$$

where $I_{0}$ denotes the intensity of the external beams at the surface of the coupler, $\alpha_{\text {ex }}$ describes the absorption coefficient, $m$ is the modulation depth and $K-$ the grating constant. For strongly absorbed external waves the interference pattern

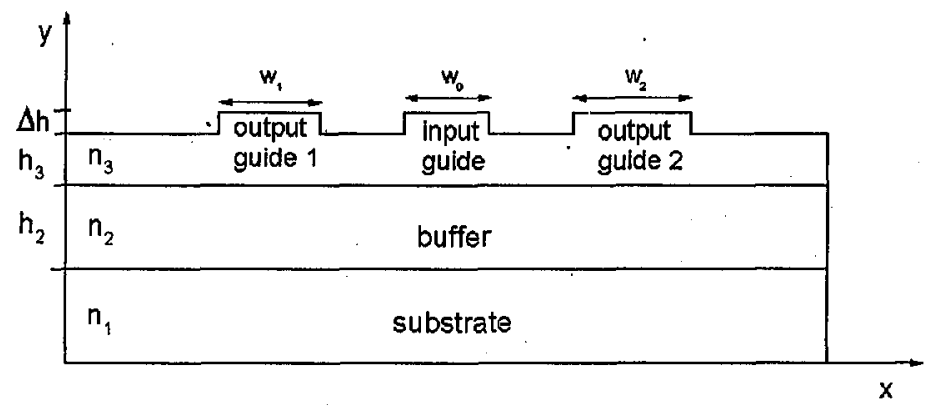

Fig. 1. Geometry of the coupler.

is created only in a thin surface layer of a depth about $1 / \alpha_{\text {ex }}$. A high frequency light excites free carriers and by the photorefractive effect leads to the modulation of refractive index. The equations describing coupling between the modes of the structure localised in the neighbouring waveguides $[6,7]$ are

$$
\begin{aligned}
& \text { i d } A_{0} / \mathrm{d} z=G_{01} A_{1} \exp \left[\mathrm{i}\left(\beta_{0}-\beta_{1}\right) z\right] f(z)+G_{02} A_{2} \exp \left[\mathrm{i}\left(\beta_{0}-\beta_{2}\right) z\right] f(z), \\
& \text { i d } A_{1} / \mathrm{d} z=G_{01} A_{0} \exp \left[-\mathrm{i}\left(\beta_{0}-\beta_{1}\right) z\right] f(z), \\
& \text { i d } A_{2} / \mathrm{d} z=G_{02} A_{0} \exp \left[-\mathrm{i}\left(\beta_{0}-\beta_{2}\right) z\right] f(z)
\end{aligned}
$$

where $f(z)=\exp (\mathrm{i} K z)+\exp (-\mathrm{i} K z)$ and $\beta_{\mu}$ are propagation constants of the modes. The modes amplitudes $A_{\mu}$ are supposed to be slowly varying functions. The coupling coefficients for TE modes are defined by

$$
G_{\mu \nu}=(\omega \varepsilon / N) \iint \Delta \varepsilon_{G} E_{\mu} E_{\nu}^{*} \mathrm{~d} x \mathrm{~d} y
$$

where $\Delta \varepsilon_{G}=2 n_{3} \Delta n_{0}$ describes the amplitude of dielectric constant changes within the grating. $N=\left(N_{\mu} N_{\nu}\right)^{1 / 2}$, where $N_{\nu}=\left(2 \beta_{\nu} / \omega \mu_{0}\right) \iint E_{\nu} E_{\nu}^{*} \mathrm{~d} x \mathrm{~d} y$ and $E_{\nu}$ is a transverse distribution of the electric field component of the $\nu$-th mode. The mismatch of the guided modes can be reduced with the help of $f(z)$ function. An analytical solution of these equations is possible in the case of a strongly mismatched coupler, when the grating induced interaction between each pair of modes can be considered separately and Eqs. (2) reduce to two independent pairs 


$$
\begin{aligned}
& \text { i } \mathrm{d} A_{0} / \mathrm{d} z=G_{01} A_{1} \exp \left[\mathrm{i}\left(\beta_{0}-\beta_{1}\right) z\right] \exp (-\mathrm{i} K z), \\
& \text { i d } A_{1} / \mathrm{d} z=G_{01} A_{0} \exp \left[-\mathrm{i}\left(\beta_{0}-\beta_{1}\right) z\right] \exp (\mathrm{i} K z),
\end{aligned}
$$

and

$$
\begin{aligned}
& \text { i d } A_{0} / \mathrm{d} z=G_{02} A_{2} \exp \left[\mathrm{i}\left(\beta_{0}-\beta_{2}\right) z\right] \exp (-\mathrm{i} K z), \\
& \text { i d } A_{2} / \mathrm{d} z=G_{02} A_{0} \exp \left[-\mathrm{i}\left(\beta_{0}-\beta_{2}\right) z\right] \exp (\mathrm{i} K z) .
\end{aligned}
$$

The first pair of equations describes coupling for grating constant $K$ close to $K_{1}=\beta_{0}-\beta_{1}$ and the second pair for $K$ close to $K_{2}=\beta_{0}-\beta_{2}$. The complete set of Eqs. (2) was solved numerically for different values of the grating constant $K$ in the case of only one waveguide excited. The relative output power in the different ports, $P_{0}=\left|A_{0}(L)\right|^{2} /\left|A_{0}(0)\right|^{2} P_{1}=\left|A_{1}(L)\right|^{2} /\left|A_{0}(0)\right|^{2}$ and $P_{2}=\left|A_{2}(L)\right|^{2} /\left|A_{0}(0)\right|^{2}$, in dependence on the normalised parameter $K / \beta_{0}$, for the propagation constants differences $\beta_{2}-\beta_{1}=\beta_{1}-\beta_{0}=0.003 \beta_{0}$, is presented in Fig. 2. The calculations have been performed for the coupling coefficients $G_{01}=G_{02}=\pi / 2 L$ (where $L$ denotes an interaction length), which enables a complete power exchange between phase matched modes. The narrow peaks in Fig. 2 illustrate the character of the couplers response to the varying grating constant and confirms the possibility of externally controlled switching between output ports. The amplitude of the refractive index grating necessary to obtain coupling can be estimated from the relation $G_{\mu \nu}=\pi / 2 L$, where the coupling coefficient dependence on the grating amplitude and the coupler parameters is given by Eq. (3).

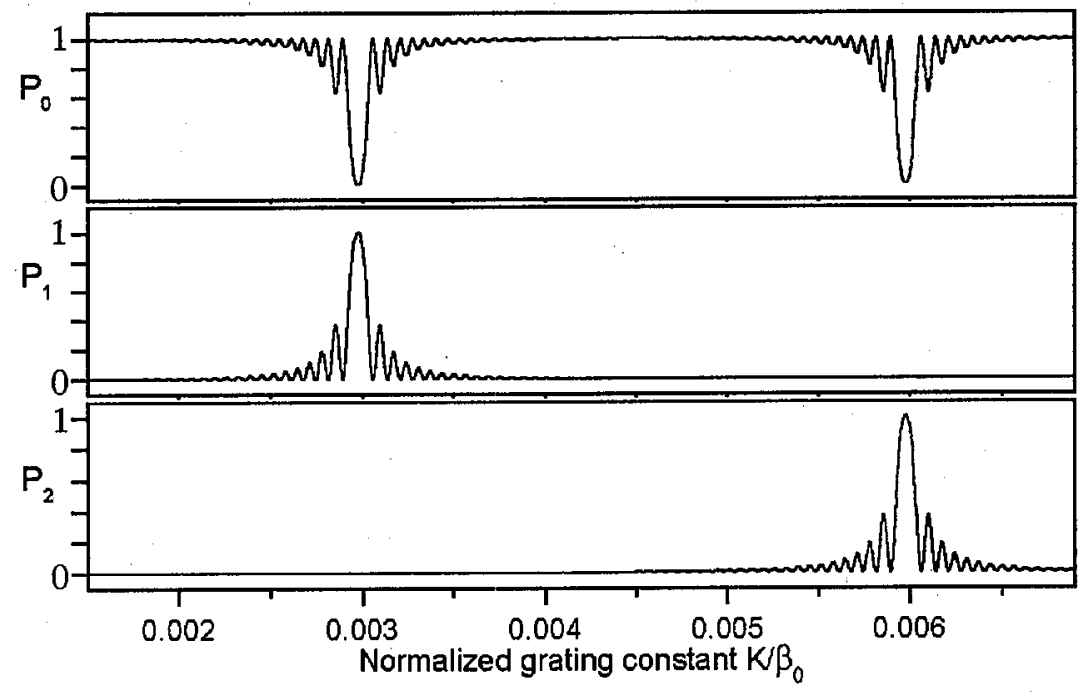

Fig. 2. The relative output power in different waveguides, $P_{0}=\left|A_{0}(L)\right|^{2} /\left|A_{0}(0)\right|^{2} P_{1}=$ $\left|A_{1}(L)\right|^{2} /\left|A_{0}(0)\right|^{2}$ and $P_{2}=\left|A_{2}(L)\right|^{2} /\left|A_{0}(0)\right|^{2}$, as functions of the normalised grating constant $K / \beta_{0}$, for the propagation constants differences $\beta_{2}-\beta_{1}=\beta_{1}-\beta_{0}=0.003 \beta_{0}$. 


\section{The semiconductor multiple quantum well device}

As an example a coupler with a guiding slab consisting of GaAs/AlGaAs multiple quantum well (MQW) layer with planes parallel to the $K$ vector of the grating was considered. The MQW structure, in accordance with previous studies $[8,9]$ is simulated by a strongly anisotropic homogeneous semiconductor with the parallel mobilities of the carriers much higher than the perpendicular ones. Parameters of the system are listed in Table. We used the effective index method

TABLE

Parameters used in this paper for $\mathrm{GaAs} / \mathrm{Al}_{0.3} \mathrm{Ga}_{0.7} \mathrm{As} \mathrm{MQW}$ layer.

\begin{tabular}{l|c|l}
\hline \hline Electron parallel mobility & $\mu_{\mathrm{e}}$ & $5000 \mathrm{~cm}^{2} \mathrm{~V}^{-1} \mathrm{~s}^{-1}$ \\
Hole parallel mobility & $\mu_{\mathrm{h}}$ & $300 \mathrm{~cm}^{2} \mathrm{~V}^{-1} \mathrm{~s}^{-1}$ \\
Average refractive index of MQW structure & $n_{3}$ & 3.5 \\
Carrier lifetime & $\tau$ & $0.2 \mathrm{~ns}$ \\
MQW thickness & $h_{3}$ & $1 \mu \mathrm{m}$ \\
Writing wavelength & $\lambda_{\mathrm{ex}}$ & $830 \mathrm{~nm}$ \\
Absorption coefficient & $\alpha_{\mathrm{ex}}$ & $10^{4} \mathrm{~cm}^{-1}$ \\
Reading wavelength & $\lambda_{\mathrm{g}}$ & $1550 \mathrm{~nm}$
\end{tabular}

and the transfer matrix approach [10] to determine both the propagation constants and field profiles of the modes. The transverse field distributions of the first three modes guided in the structure are presented in Fig. 3 . The dependence of the relative coupling coefficients, $G_{\mu \nu} / \Delta \varepsilon_{G}$, on the radius and position of the external waves is shown in Figs. 4a,b. The transverse distribution of the external waves intensities, $I_{0}(x)$, is assumed to have a shape of Gaussian beam focused at the surface of the coupler. The change of $G_{\mu \nu}$ from positive to negative values in dependence on the position of the external beams is due to the different numbers of knots in the transverse electric field distribution of particular modes.

For a given device length $L$ the coupling coefficient needs to be $G_{\mu \nu}=$ $\pi / 2 L$ to yield complete switching. Knowing $G_{\mu \nu}$ one can calculate the modulation amplitude of the grating. The grating amplitude and fringes spacing necessary for transfer signals to the first output port of $1 \mathrm{~cm}$ long device are $\Delta n_{01}=5 \times 10^{-4}$ and $A_{1}=137 \mu \mathrm{m}$ while in the case of the second output port $\Delta n_{02}=4 \times 10^{-4}$ and $\Lambda_{2}=246 \mu \mathrm{m}$.

For high frequency external waves the main photorefractive mechanism in semiconductor MQW layer relies on interband excitation of electrons and holes, their movement due to the drift and diffusion and finally a recombination to the donor traps. The resulting space-charge electric field cause the change of the refractive index through the electrooptic effect. Such model leads to the following equations based on a classical Kukhtarev-Vinetskii model [8]:

$$
\frac{\partial n_{\mathrm{e}}}{\partial t}=\frac{\alpha}{h \nu} I-\gamma_{\mathrm{e}} n_{\mathrm{e}} N_{\mathrm{D}}^{+}+\frac{1}{e} \frac{\partial j_{\mathrm{e}}}{\partial z},
$$




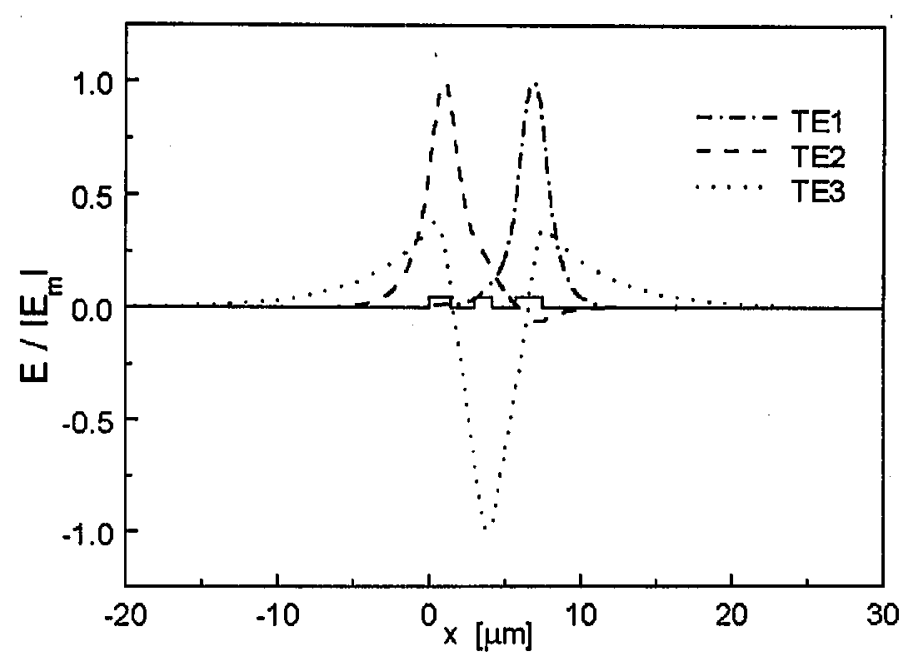

Fig. 3. The transverse field distributions of the first three TE modes guided in the structure presented in Fig. 1 for waveguides heights $\Delta h_{0}=0.1 \mu \mathrm{m}, \Delta h_{1}=0.2 \mu \mathrm{m}$, $\Delta h_{2}=0.3 \mu \mathrm{m}$ and $\Delta h_{3}=1 \mu \mathrm{m}$, waveguides widths $w_{0}=1.1 \mu \mathrm{m}, w_{1}=1.4 \mu \mathrm{m}$, $w_{2}=1.7 \mu \mathrm{m}$, and waveguides separation $1.6 \mu \mathrm{m}$. Refractive index of the MQW layer $n_{3}=3.5$ and of the substrate $n_{2}=2.9$, the wavelength of guided modes $\lambda_{\mathrm{g}}=1.55 \mu \mathrm{m}$. The TE1, TE2 and TE3 modes are marked in the text by subscripts $\mu=2, \mu=1$, and $\mu=0$ respectively.

$$
\begin{aligned}
& \frac{\partial n_{\mathrm{h}}}{\partial t}=\frac{\alpha}{h \nu} I-\gamma_{\mathrm{h}} n_{\mathrm{h}}\left(N_{\mathrm{D}}-N_{\mathrm{D}}^{+}\right)-\frac{1}{e} \frac{\partial j_{\mathrm{h}}}{\partial z}, \\
& j_{\mathrm{e}}=e \mu_{\mathrm{e}} n_{\mathrm{e}} E+\mu_{\mathrm{e}} k_{\mathrm{B}} T \frac{\partial n_{\mathrm{e}}}{\partial z} \\
& j_{\mathrm{h}}=e \mu_{\mathrm{h}} n_{\mathrm{h}} E-\mu_{\mathrm{h}} k_{\mathrm{B}} T \frac{\partial n_{\mathrm{h}}}{\partial z}, \\
& \frac{\partial N_{\mathrm{D}}^{+}}{\partial t}=\gamma_{\mathrm{h}} n_{\mathrm{h}} N_{\mathrm{D}}^{0}-\gamma_{\mathrm{e}} n_{\mathrm{e}} N_{\mathrm{D}}^{+}, \\
& \frac{\partial E}{\partial z}=\frac{e}{\varepsilon_{0}}\left(N_{\mathrm{D}}^{+}+n_{\mathrm{h}}-n_{\mathrm{e}}-N_{\mathrm{A}}\right),
\end{aligned}
$$

The symbols in the above equations are: $n_{\mathrm{e}}-$ the free electron concentration, $n_{\mathrm{h}}$ - the free hole concentration, $N_{\mathrm{D}}$ - the concentration of donors, $N_{\mathrm{D}}^{+}-$the concentration of ionised donors, $N_{\mathrm{A}}$ - the concentration of acceptors, $j_{\mathrm{e}}$ - the electronic current density, $j_{\mathrm{h}}-$ the hole current density, $E-$ the total electric field ( $E=E_{0}+E_{\mathrm{sc}}$, where $E_{\mathrm{sc}}$ is a space-charge field and $E_{0}$ is an external field), $I$ - the light intensity, $\gamma_{\mathrm{e}}$ - the constant describing recombination of electrons, $\gamma_{\mathrm{h}}-$ the constant describing recombination of holes, $\mu_{\mathrm{e}}-$ electron mobility along the quantum wells, $\mu_{\mathrm{h}}$ - hole mobility along the quantum wells, $\varepsilon_{0}$ - the permittivity of the vacuum, $\varepsilon$ - the effective dielectric constant of MQW structure, $e$ - the absolute value of the elementary charge, $k_{\mathrm{B}}$ - the Boltzmann 

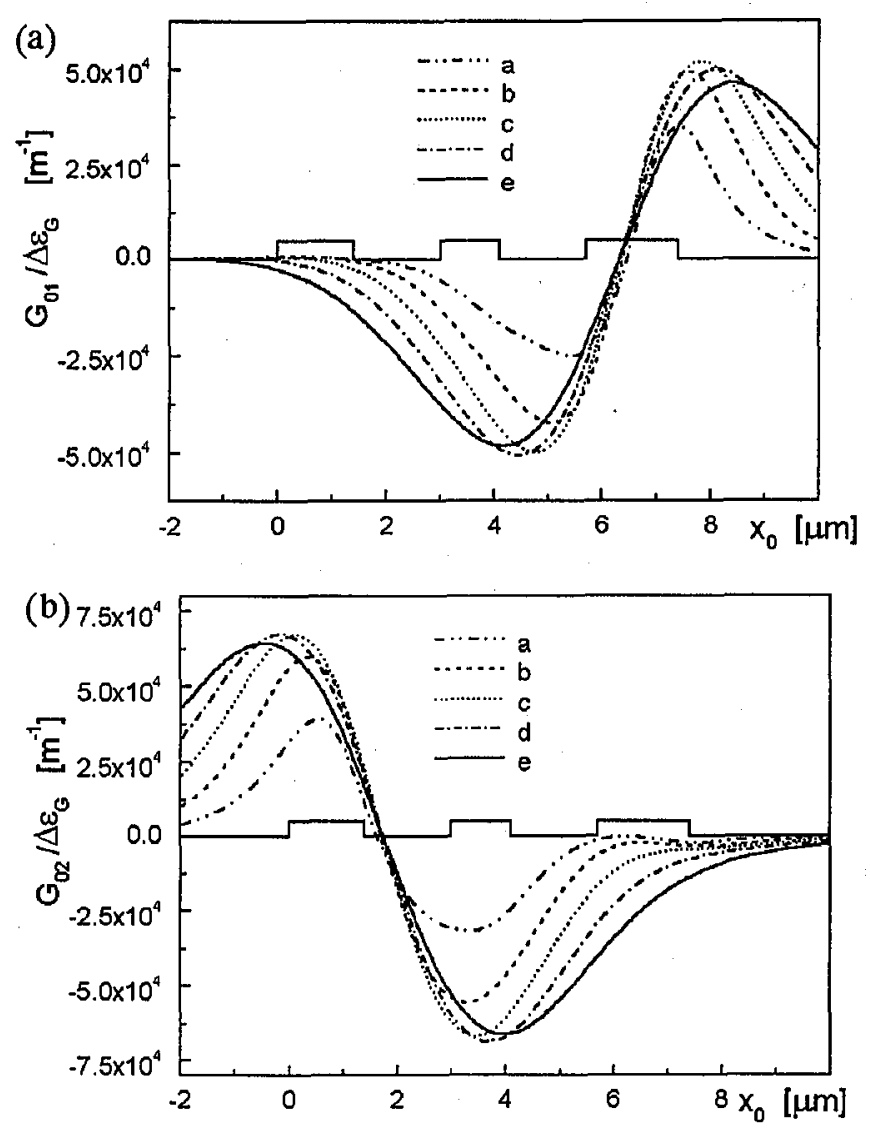

Fig. 4. (a) The dependence of the normalised coupling coefficient $G_{01} / \Delta \varepsilon_{G}$ on the position of the external beams for different radii: $a-\rho=0.5 \mu \mathrm{m}, b-\rho=1 \mu \mathrm{m}, c-$ $\rho=1.5 \mu \mathrm{m}, d-\rho=2 \mu \mathrm{m}, e-\rho=2.5 \mu \mathrm{m}$. (b) The dependence of the normalised coupling coefficient $G_{02} / \Delta \varepsilon_{G}$ on the position of the external beams for different radii: $a-\rho=0.5 \mu \mathrm{m}, b-\rho=1 \mu \mathrm{m}, c-\rho=1.5 \mu \mathrm{m}, d-\rho=2 \mu \mathrm{m}, e-\rho=2.5 \mu \mathrm{m}$.

constant and $T$ - the absolute temperature. Thermal excitation of the carriers and transverse carrier mobility are not included in the above set of equations. The latter condition is fulfilled due to the high difference between longitudinal and transverse carrier mobilities characteristic of multiple quantum wells systems. The refractive index change in the semiconductor MQW structure $[11,12]$ depends on total electric field, $\Delta n(E)=(-1 / 2) n_{3}^{3}\left(r E+s E^{2}\right)$, where $r$ denotes linear and $s$ quadratic electrooptic coefficient. For the wavelengths far from the resonance the linear effect (with $r_{41}=1 \mathrm{pm} / \mathrm{V}$ for bulk GaAs) dominates. In the resonant case the quadratic electrooptic coefficient increase up to $s=2 \times 10^{-12} \mathrm{~cm}^{2} / \mathrm{V}^{2}$ at the wavelength of the heavy hole excitons absorption [11].

The set of Eqs. (6) was solved numerically for the initial conditions $E(0)=0$ and $N_{\mathrm{D}}^{+}(0)=N_{\mathrm{A}}=N_{\mathrm{D}} / 2$ and material parameters listed in Table. The time 
evolution of the space-charge field modulation amplitude for different intensities of the external waves is presented in Fig. 5. The calculations predict the same saturation value of the space-charge field for different light intensities and a strong influence of intensity on the response time of the device. The steady state value of the space-charge field can be increased by a proper choice of the trap concentra-

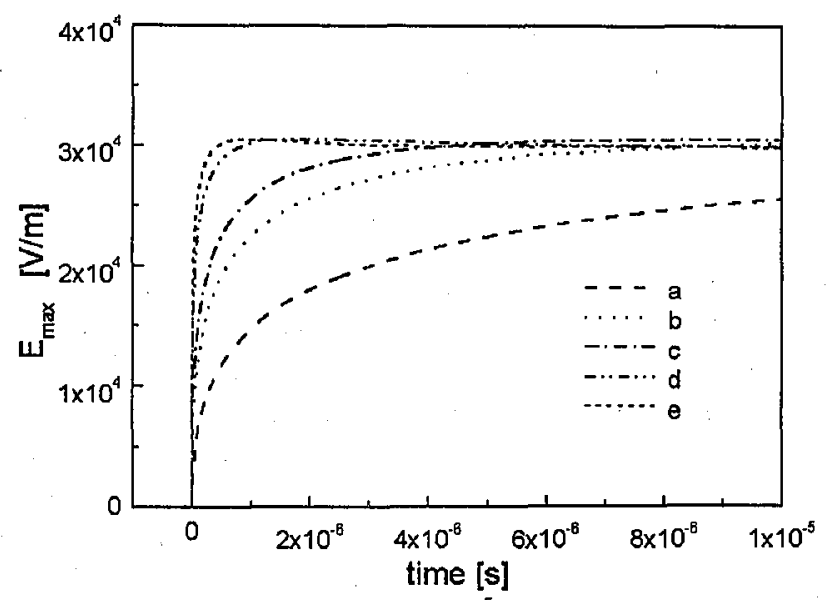

Fig. 5. The time evolution of the space-charge field amplitude for different intensities of the external waves: $a-I_{0}=10^{3} \mathrm{~W} / \mathrm{m}^{2}, b-I_{0}=5 \times 10^{3} \mathrm{~W} / \mathrm{m}^{2}, c-I_{0}=10^{4} \mathrm{~W} / \mathrm{m}^{2}$, $d-I_{0}=5 \times 10^{4} \mathrm{~W} / \mathrm{m}^{2}, e-I_{0}=10^{5} \mathrm{~W} / \mathrm{m}^{2}$ and $N_{\mathrm{D}}=10^{23} \mathrm{~m}^{-3}$. The material parameters used for calculations are listed in Table.

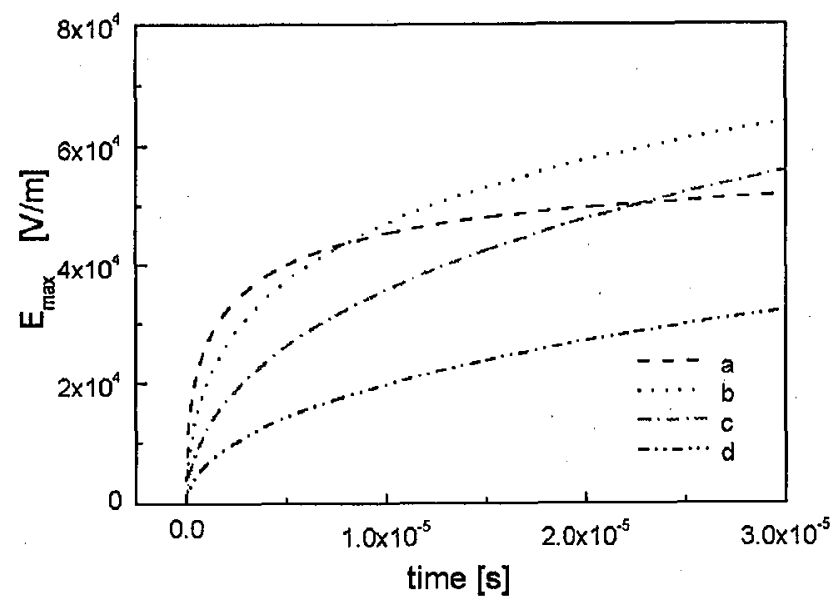

Fig. 6. The time evolution of the space-charge field amplitude for $I_{0}=10^{2} \mathrm{~W} / \mathrm{m}^{2}$ and different values of trap densities: $a-N_{\mathrm{D}}=3 \times 10^{23} \mathrm{~m}^{-3}, b-N_{\mathrm{D}}=10^{24} \mathrm{~m}^{-3}$, $c-N_{\mathrm{D}}=3 \times 10^{24} \mathrm{~m}^{-3}, d-N_{\mathrm{D}}=10^{25} \mathrm{~m}^{-3}$. The initial conditions and material parameters are the same as in Fig. 5. 
tion. The influence of $N_{\mathrm{D}}$ value on the space-charge field evolution is illustrated in Fig. 6 . The obtained results suggest the necessity of higher sensitivity system. The steady value of space charge field can be increased either by applying an external electric field or by changing the material. The properties of MQW coupler with the longitudinal and transverse external electric fields will be a subject of our future research.

The grating lifetime depends mainly on the materials dark conductivity and in the absence of free carriers which is assumed in the presented model $\left(N_{\mathrm{D}}^{+}(0)=\right.$ $N_{\mathrm{A}}=N_{\mathrm{D}} / 2$ ) can be very long, allowing for recording of the grating by the pulses of the external waves.

\section{Conclusions}

It has been shown that a thin photorefractive grating can provide coupling between different guides of the asymmetric three channel directional coupler. A choice of the output guide, the efficiency of coupling and the wavelength of the switched signals depend on the grating parameters and can be varied during the work of the device. The grating does not require permanent presence of the external waves. Pump pulses are necessary only for writing, refreshing or erasing of the grating. Signal waves at low frequency do not destroy the grating which enables a potential application of the system as an all-optical switching element with memory. The switching times of the MQW coupler considered as an example are in the range of microseconds.

\section{References}

[1] C. Elachi, C. Yeh, Opt. Commun. 7, 201 (1973).

[2] D. Marcuse, J. Lightwave Technol. LT-5, 268 (1987).

[3] W.P. Huang, H.A. Haus, J. Lightwave Technol. LT-7, 920 (1989).

[4] G. Griffel, A. Yariv, IEEE J. Quantum Electron. QE-27, 1115 (1991).

[5] R.R.A. Syms, J. Opt. Soc. Am. A 8, 1062 (1991).

[6] E. Weinert-Rączka, J. Opt. Soc. Am. B 11, 2340 (1994).

[7] E. Weinert-Rączka, W. Biehlig, F. Lederer, J. Opt. Soc. Am. B 14, 3232 (1997).

[8] L.F. Magaña, F. Agulló-López, M. Carrascosa, J. Opt. Soc. Am. B 11, 1651 (1994).

[9] M. Aguilar, M. Carrascosa, F. Agulló-López, J. Opt. Soc. Am. B 13, 2630 (1996).

[10] F. Lederer, U. Trutschel, C. Wächter, J. Opt. Soc. Am. A 8, 1536 (1991).

[11] D.D. Nolte, D.H. Olson, G.E. Doran, W.H. Knox, A.M. Glass, J. Opt. Soc. Am. $B$ 7, 2217 (1990).

[12] Q. Wang, R.M. Brubaker, D.D. Nolte, M.R. Melloch, J. Opt. Soc. Am. B 9, 1626 (1992). 